\title{
THE ASSOCIATIVE FORMS OF THE GRADED CARTAN TYPE LIE ALGEBRAS
}

\author{
ROLF FARNSTEINER
}

\begin{abstract}
This paper determines the Cartan type Lie algebras that possess a nonsingular associative form.
\end{abstract}

0. Introduction. The structure of Cartan type Lie algebras has been extensively studied for several years. Derivation algebras and isomorphism classes of Cartan subalgebras are well known and it is the objective of the present paper to determine those graded Cartan type Lie algebras which possess a nonsingular associative form.

In contrast with trace forms, general nonsingular associative forms do not play an eminent role in the classification theory of simple Lie algebras of prime characteristic. Their significance primarily rests on cohomology theory. Nonsingular associative forms allow the study of central extensions of Lie algebras by means of derivations. We will employ a slight generalization of this known interrelation in a forthcoming paper.

The introductory remarks of the first section are followed by a brief investigation of Cartan subalgebras of graded Lie algebras. Our results generalize those of $\mathrm{M}$. Frank (cf. [8]). \$3 establishes general properties of associative forms of graded simple Lie algebras and provides the basic tools for the study of the graded Cartan type Lie algebras.

The author would like to express his gratitude to Professor Helmut Strade for many helpful suggestions as well as to the referee for proposing Proposition 3.4 and the resulting simplifications in \$4.

1. Graded representations of graded Lie algebras. In the sequel, $L=\oplus_{i=-r}^{k} L_{i}, r$, $k \geqslant 1$, denotes a graded Lie algebra over an arbitrary field $F$. A representation $T$ : $L \rightarrow \operatorname{gl}(V)$ is said to be graded if $V=\oplus_{j=-s}^{l} V_{j}$ and $T(x)\left(V_{j}\right) \subset V_{i+j}$ for every $x \in L_{i}$ and $-s \leqslant j \leqslant l$. Note that each of the $V_{j}$ is an $L_{0}$-module. We will always assume that $l, s \geqslant 1$. We consider the subalgebras $L^{+}:=\bigoplus_{i=1}^{k} L_{i}$ and $L^{-}:=\bigoplus_{i=-r}^{-1} L_{i}$. Using the P-B-W Theorem it can be readily verified that we have $U(L)=U\left(L^{-}\right) U\left(L_{0}\right) U\left(L^{+}\right)=U\left(L^{+}\right) U\left(L_{0}\right) U\left(L^{-}\right)$for the respective universal enveloping algebras.

Received by the editors May 17, 1984 and, in revised form, April 10, 1985.

1980 Mathematics Subject Classification. Primary 17B20. 
Lemma 1.1. Let $V=\bigoplus_{j=-s}^{l} V_{j}$ be a graded L-module. Suppose that $W \subset V_{j}$ is a subspace. Then

$$
U\left(L^{+}\right) W \subset W+L_{1} W+\sum_{i=j+2}^{l} V_{i} \text { and } U\left(L^{-}\right) W \subset W+L_{-1} W+\sum_{i=-s}^{j-2} V_{i} .
$$

Proof. Put $X:=W+L_{1} W+\sum_{i=j+2}^{\prime} V_{i}$. It is clear that $x \cdot X \subset X$ for every $x \in L^{+}$. This shows that $X$ is $U\left(L^{+}\right)$-invariant. Let $A$ be the set of all $x$ in $U\left(L^{+}\right)$ such that $x \cdot W \subset X$. By the above, $A$ is a subalgebra of $U\left(L^{+}\right)$containing $L^{+}$. Consequently, $A=U\left(L^{+}\right)$and $U\left(L^{+}\right) W \subset X$. The proof of the second assertion is completely analogous.

Proposition 1.2. Suppose that $V=\bigoplus_{j=-s}^{\prime} V_{j}$ is an irreducible L-module. Then the following statements hold:

(1) $V_{-s}$ and $V_{l}$ are irreducible $L_{0}$-modules.

(2) $V_{-s+1}=L_{1} V_{-s}, V_{l-1}=L_{-1} V_{l}$.

(3) $L_{0} V_{-s}=V_{-s}, L_{0} V_{l}=V_{l}$.

Proof. Let $W$ be a nonzero $L_{0}$-module of $V_{-s}$. Consider the subspace $X:=\sum_{i=0}^{k} L_{i} W$. As $L_{j} W=0$ for $j<0$ we see that $U\left(L_{0}\right) U\left(L^{-}\right) X \subset X$. If $X=0$ we obtain $L \cdot W=0$ and the irreducibility of $V$ entails $V=W=V_{-s}$ which contradicts our general assumption pertaining to $l$. By 1.1 we obtain

$$
V=U(L) X=U\left(L^{+}\right) X=L_{0} W+L_{1} W+\sum_{i=-s+2}^{l} V_{i}
$$

This proves $V_{-s}=L_{0} W \subset W$ as well as $L_{1} W=V_{-s+1}$. Hence properties (1)-(3) follow and the assertions pertaining to $V_{l}$ are obtained analogously.

In the sequel we are going to apply the above results to the situation where $V$ equals $L$.

Definition. Let $L=\oplus_{i=-r}^{k} L_{i}$ be a graded Lie algebra. $L$ is said to be admissibly graded if $L_{-r}=C_{L}\left(L_{-1}\right)$, where $C_{L}\left(L_{-1}\right)$ denotes the centralizer of $L_{-1}$ in $L$.

Corollary 1.3. Suppose that $L$ is simple and such that $\left[L_{-1}, L_{i}\right]=L_{i-1}$ for $i \leqslant-1$. Then $L$ is admissibly graded.

Proof. Consider the graded $L_{0}$-submodule $V=C_{L}\left(L_{-1}\right)$ of $L$. We propose to verify that $V_{i}:=V \cap L_{i}$ is $U\left(L^{-}\right)$-invariant. By definition, we have $L_{-1} V_{i}=0$. Suppose that for some $j \leqslant-1$ the equation $L_{j} V_{i}=0$ holds for $-r \leqslant i \leqslant k$. Then

$$
L_{j-1} V_{i}=\left[L_{-1}, L_{j}\right] \cdot V_{i} \subset L_{-1} \cdot\left(L_{j} V_{i}\right)+L_{j} \cdot\left(L_{-1} V_{i}\right)=0 \text {. }
$$

This proves by induction that $L_{j} V_{i}=0$ for $j \leqslant-1$ and $-r \leqslant i \leqslant k$. Hence $V_{i}$ is $U\left(L^{-}\right)$-invariant and in the case of $V_{i}$ being nonzero we obtain $L=U(L) V_{i}=$ $U\left(L^{+}\right) V_{i} \subset V_{i}+\sum_{j=i+1}^{k} L_{j}$. Hence $i=-r$ and $V=V_{-r}=L_{-r}$.

REMARK. It is noteworthy that the condition $\left[L_{-1}, L_{i}\right]=L_{i-1}, i \leqslant-1$, is automatically fulfilled in the case $r \leqslant 2$. If $r$ equals 2 , we can, by virtue of 1.2 only have $\left[L_{-1}, L_{-1}\right]=L_{-2}$ or $\left[L_{-1}, L_{-1}\right]=0$. The latter alternative implies $L=U(L) L_{-1}=$ $U\left(L^{+}\right) L_{-1} \subset \sum_{j=-1}^{k} L_{j}$, a contradiction. As a result, every simple, graded Lie algebra $L=\oplus_{i=-r}^{k} L_{i}$ with $r \leqslant 2$ is admissibly graded. 
2. Cartan subalgebras of graded Lie algebras. In this section we will generalize a result attributable to $M$. Frank that deals with Cartan subalgebras of graded Lie algebras. Our Theorem 2.2 will be useful in the study of certain Cartan subalgebras of the Lie algebras of Cartan type.

THEOREM 2.1. Let $L=\oplus_{i=-r}^{k} L_{i}$ be a graded Lie algebra of finite dimension. Then the following statements hold:

(1) If $H$ is a Cartan subalgebra of $L_{0}$, then

$$
\Omega=\left\{x \in L ; \text { for every } h \in H \text { there is } n(h) \in N: \operatorname{ad}_{h}^{n(h)}(x)=0\right\}
$$

is a graded Cartan subalgebra of $L$.

(2) If $L$ is restricted and centerless, then $L_{0}$ is a p-subalgebra of $L$ and every maximal torus $T$ of $L_{0}$ is a maximal torus of $L$.

Proof. (1) Note that $\Omega$ is the Fitting 0 -component of $L$ relative to $H$. We now apply Theorem 1, p. 135 of [14] in order to see that $\Omega$ is a Cartan subalgebra of $L$. It is easy to see that $\Omega=\oplus_{i=-r}^{k} \Omega \cap L_{i}$.

(2) As $L$ is centerless, we have $L_{j}^{[p]} \subset L_{p j}$ for every integer $j$. We consider $\Omega:=C_{L}(T)$. Note that $\Omega$ is graded. Let $x$ be an element of $\Omega_{0}$. Then there exists a positive integer $m$ such that $x^{[p]^{m}}$ is semisimple. Consequently, $x^{[p]^{m}}$ is contained in $T$ and $\operatorname{ad}_{x}^{p^{m}}(\Omega)=0$. This shows that $\Omega_{0}$ acts nilpotently on $\Omega$. We now apply the Lemma on p. 134 of [14] in order to see that $\Omega$ is nilpotent.

Note that $\Omega$ is a $p$-subalgebra with $\Omega_{j}^{[p]} \subset \Omega_{p j}$. The center $C(\Omega)$ is also graded. Let $x$ be a semisimple element of $\Omega$. As $\Omega$ is nilpotent, $x$ is central in $\Omega$ and we write $x=\sum_{i=-r}^{k} x_{i}$ where the elements $x_{i}$ are contained in $C(\Omega) \cap \Omega_{i}$. Hence $x^{[p]^{n}}=\sum_{i=-r}^{k} x_{i}^{[p]^{n}}$ for every natural number $n$. Consequently, there is $m$ such that $x^{[p]^{m}}=x_{0}^{[p]^{m}}$. As $x$ is semisimple, we obtain $x \in \Omega_{0}$. The maximality of $T$ now implies that $x$ is contained in $T$.

Let $T_{1}$ be a maximal torus of $L$ containing $T$. Then $T_{1} \subset \Omega$ and our above considerations show that $T_{1} \subset T$. This concludes the proof of our theorem.

Let $V$ be a finite dimensional vector space over $F$. We call an endomorphism $f$ : $V \rightarrow V$ semisimple if it becomes diagonable after some base field extension.

The following result is a generalization of [8, Theorem 1.4].

THEOREM 2.2. Supose that $L=\oplus_{i=-r}^{k} L_{i}, r, k \geqslant 1$, is a finite dimensional admissibly graded Lie algebra such that $\left[L_{-1}, L_{i}\right]=L_{i-1}$ for $i \leqslant-1$. Let $H \subset L_{0}$ be a Cartan subalgebra such that $\mathrm{ad}_{h}: L_{-1} \rightarrow L_{-1}$ is semisimple for every $h \in H$. Then the following statements hold:

(1) $H$ is abelian.

(2) $\mathrm{ad}_{h}$ is semisimple for every $h$ in $H$.

(3) The centralizer $C_{L}(H)$ is a Cartan subalgebra of $L$.

Proof. (1) Consider the representation $\Gamma: L_{0} \rightarrow \mathrm{gl}\left(L_{-1}\right)$ which is induced by the adjoint representation. Then $\Gamma(H)$ is an ad-semisimple Lie algebra in the sense of [6]. Hence $\Gamma(H)$ is abelian. Since $L$ is admissibly graded, $\Gamma$ is faithful. Consequently, $H$ is abelian. 
(2) Suppose first that $F$ is algebraically closed. Then $\Gamma(H)$ is simultaneously diagonable. Consider the root space decomposition $L=\bigoplus_{\alpha \in R} L^{(\alpha)}$ of $L$ relative to $H$. We obtain $L_{i}=\oplus_{\alpha \in R} L^{(\alpha)} \cap L_{i}$ and $L^{(0)} \cap L_{0}=H$. According to $2.1, \Omega=$ $\oplus_{i=-r}^{k} L^{(0)} \cap L_{i}$ is a Cartan subalgebra of $L$. Our assumption implies

$$
L^{(\alpha)} \cap L_{-1}=\left\{x \in L_{-1} ;[h, x]=\alpha(h) x \text { for every } h \in H\right\} .
$$

We are going to show inductively that

$$
L^{(\alpha)} \cap L_{i}=\left\{x \in L_{i} ;[h, x]=\alpha(h) x \text { for every } h \in H\right\}
$$

for every $i$. Suppose that $(*)$ holds for some $i \geqslant-1$. Let $x$ be an element of $L^{(\alpha)} \cap L_{-1}$ for some $\alpha \in R \cup\{0\}$. Suppose that $y$ is an element of $L^{(\beta)} \cap L_{i+1}$. Then $[x, y] \in L^{(\alpha+\beta)} \cap L_{i}$ and we obtain for $h \in H$

$$
\begin{aligned}
{[x,[h, y]-\beta(h) y] } & =[x,[h, y]]-\beta(h)[x, y] \\
& =[[x, h], y]+[h,[x, y]]-\beta(h)[x, y] \\
& =-\alpha(h)[x, y]+(\alpha+\beta)(h)[x, y]-(\beta)(h)[x, y]=0 .
\end{aligned}
$$

Since $L$ is admissibly graded the above yields $[h, y]=\beta(h) y$. This proves $(*)$ for $-1 \leqslant i \leqslant k$.

Suppose that $(*)$ is valid for some $i \in\{-r+1, \ldots,-1\}$. Then the equation $L_{i-1}=\left[L_{-1}, L_{i}\right]$ entails

$$
L^{(\alpha)} \cap L_{i-1}=\sum_{\beta+\gamma=\alpha}\left[L^{(\beta)} \cap L_{-1}, L^{(\gamma)} \cap L_{i}\right]
$$

which shows that $(*)$ is valid for $i-1$. We have therefore proved (2). In the general case, let $\bar{F}$ be an algebraic closure of $F$ and consider $\bar{L}:=L \otimes_{F} \bar{F}$ as well as $\bar{H}:=H \otimes_{F} \bar{F}$. It is easily seen that the pair $(\bar{L}, \bar{H})$ fulfills the conditions of the theorem and we obtain (2).

Assertion (3) is an immediate consequence of (2) and 2.1.

3. Associative forms on simple graded Lie algebras. Let $L$ be a Lie algebra over $F$ and let $V \subset L$ be an $F$-subspace. A symmetric bilinear form $f: L \times L \rightarrow F$ is called $V$-associative if $f([x, v], y)=f(x,[v, y]) \forall x, y \in L \forall v \in V$. An $L$-associative form is referred to as associative.

We recall some basic facts concerning associative forms. The subspace $\operatorname{rad}(f):=\{x \in L ; f(x, y)=0 \forall y \in L\}$ is an ideal of $L$, called the radical of $f$. If $L$ is simple, then either $f=0$ or $f$ is nonsingular, i.e. $\operatorname{rad}(f)=0$. Suppose that $L$ is finite dimensional and that $L=\bigoplus_{\alpha \in R} L_{\alpha}$ is a root space decomposition of $L$ relative to a nilpotent subalgebra $H$. Then $f\left(L_{\alpha}, L_{\beta}\right)=0$ for $\alpha, \beta \in R$ and $\alpha+\beta \neq 0$. If $f$ is nonsingular, so is its restriction to $L_{\alpha} \times L_{-\alpha} \forall \alpha \in R$. The latter fact is frequently referred to by saying that $L_{\alpha}$ and $L_{-\alpha}$ are dually paired.

We finally note that given a symmetric bilinear form $f$ the set $H:=\{z \in L$ : $f([x, z], y)=f(x,[z, y]) \forall x, y \in L\}$ is a subalgebra of $L$.

Throughout this section $L=\oplus_{i=-r}^{k} L_{i}, k, r \geqslant 1$, is assumed to be a finite dimensional simple graded Lie algebra over $F$.

Proposition 3.1. Suppose that $\lambda: L \times L \rightarrow F$ is an associative form. Then we have $\lambda\left(L_{i}, L_{j}\right)=0$ for $i+j \neq k-r$. 
Proof. As $\operatorname{rad}(\lambda)$ is an ideal of $L$, we either have $\lambda=0$ or $\operatorname{rad}(\lambda)=0$. In the latter case $\lambda$ induces an isomorphism $\psi: L \rightarrow L^{*}$ of $L$-modules. As $L^{*}$ is graded by $\left(L^{*}\right)_{i}:=\left\{f \in L^{*} ; f\left(L_{j}\right)=0\right.$ for $\left.j \neq-i\right\}, \operatorname{Hom}_{L}\left(L, L^{*}\right)$ is graded and we write $\psi=\sum_{i} \psi_{i}, \operatorname{deg}\left(\psi_{i}\right)=i$. Let $j$ be an index such that $\psi_{j} \neq 0$. Then $\operatorname{ker} \psi_{j}$ is properly contained in $L$ and the simplicity of $L$ enforces $\psi_{j}$ to be injective. Hence $\psi_{j}\left(L_{l}\right) \neq 0$ for $l=-r, k$. As $L^{*}=\oplus_{i=-k}^{r}\left(L^{*}\right)_{i}$ this implies $-k \leqslant j-r, j+k \leqslant r$. Consequently, $j=r-k$ and $\psi=\psi_{r-k}$.

Let $x, y$ be elements of $L_{i}, L_{j}$, respectively. Since $\psi$ is defined by $\lambda$, we obtain $\lambda(x, y)=\psi(x)(y)$ with $\psi(x) \in\left(L^{*}\right)_{i+r-k}$. This shows that $\lambda(x, y)=0$ if $j \neq-i$ $+k-r$.

COROLlARY 3.2. Suppose that $H \subset L_{0}$ is a nilpotent subalgebra of $L$ with root space decomposition $L=\oplus_{\alpha \in R} L_{\alpha}$ and assume that $\lambda: L \times L \rightarrow F$ is a nonsingular associative form. Then the following statements hold:

(1) If $H$ is a Cartan subalgebra of $L$, then $r=k$.

(2) $\lambda$ is nonsingular on $L_{i} \times L_{j}$ for $i+j=k-r$ and $\operatorname{dim}_{F} L_{i}=\operatorname{dim}_{F} L_{k-r-i}$.

(3) $\lambda$ is nonsingular on $L_{i} \cap L_{\alpha} \times L_{j} \cap L_{-\alpha}$ for $i+j=k-r$ and $\operatorname{dim}_{F} L_{i} \cap L_{\alpha}$ $=\operatorname{dim}_{F} L_{k-r-i} \cap L_{-\alpha}$ for every $\alpha \in R$.

Proof. (1) As $\lambda$ is nonsingular and $H$ is a Cartan subalgebra, we have $\lambda(H, H)$ $\neq 0$. Thus $0=k-r$ by 3.1 .

(2) This follows directly from 3.1.

(3) Since $H$ is contained in $L_{0}$, we obtain a decomposition

$$
L=\bigoplus_{i=-r}^{k} \bigoplus_{\alpha \in R} L_{i} \cap L_{\alpha} .
$$

Our assertion now follows from (2) and our introductory remarks.

COROllaRY 3.3. Suppose that $H \subset L_{0}$ is a Cartan subalgebra of $L_{0}$. If $L$ possesses a nonsingular trace form, then $H$ is a Cartan subalgebra of $L$ and $k=r$.

Proof. By virtue of Theorem 2.1 there is a graded Cartan subalgebra $\Omega$ containing $H$. Since $L$ has a nonsingular trace form, $\Omega$ operates on $L$ by semisimple endomorphisms (cf. [2]). Consequently, $\Omega_{i}=0$ for $i \neq 0$, thus $\Omega=H$. We now apply the preceding result in order to see that $k=r$.

Let $S: U(L) \rightarrow U(L)$ denote the antipode map on $U(L)$, i.e. the antihomomorphism on $U(L)$ satisfying $S(1)=1$ and $S(x)=-x \forall x \in L$.

Proposition 3.4. Suppose that there is a nondegenerate $L_{0}$-associative bilinear form $f$ on $L_{-r} \times L_{k}$. Extend $f$ to $f_{1}: L \times L_{k} \rightarrow F$ by $f_{1}\left(L_{i}, L_{k}\right)=0, i \neq-r$. Assume that for any $u \in U\left(L^{-}\right), v, w \in L_{k}$ we have

$$
f_{1}(u v, w)=f_{1}(S(u) w, v) .
$$

Then $f_{1}$ extends to a nondegenerate symmetric associative bilinear form $\lambda$ on $L \times L$.

Proof. Note that $f_{1}$ is $L_{0}$-associative and that

$$
f_{1}(S(u) v, w)=f_{1}(v, u w) \quad \forall u \in U\left(L_{0}\right) .
$$


We first show that if $u, u^{\prime} \in U\left(L^{-}+L_{0}\right), v \in L_{k}$ and $u v=0$, then

$$
f_{1}\left(S(u) u^{\prime} v, v\right)=0 .
$$

As $U\left(L^{-}+L_{0}\right)=U\left(L^{-}\right) U\left(L_{0}\right)$ and as (2) is linear in $u^{\prime}$ it is sufficient to prove this in case $u=\sum_{i} u_{-}^{(i)} u_{0}^{(i)}, u^{\prime}=u_{-}^{\prime} u_{0}^{\prime}$, where $u_{-}^{(i)}, u_{-}^{\prime} \in U\left(L^{-}\right)$and $u_{0}^{(i)}, u_{0}^{\prime} \in U\left(L_{0}\right)$. Then

$$
\begin{aligned}
f_{1}\left(S(u) u^{\prime} v, v\right) & =\sum_{i} f_{1}\left(S\left(u_{0}^{(i)}\right) S\left(u_{-}^{(i)}\right) u_{-}^{\prime} u_{0}^{\prime} v, v\right) \\
& =\sum_{i} f_{1}\left(S\left(u_{-}^{(i)}\right) u_{-}^{\prime} u_{0}^{\prime} v, u_{0}^{(i)} v\right) \quad(\text { by }(1)) \\
& =\sum_{i} f_{1}\left(S\left(u_{-}^{\prime}\right) u_{-}^{(i)} u_{0}^{(i)} v, u_{0}^{\prime} v\right) \quad(\text { by }(*)) \\
& =f_{1}\left(S\left(u_{-}^{\prime}\right) u v, u_{0}^{\prime} v\right)=0 .
\end{aligned}
$$

Fix a nonzero element $v \in L_{k}$. The results of $\S 1$ entail $L=U\left(L^{-}+L_{0}\right) \cdot v$ and we put

$$
\lambda\left(u^{\prime} v, u v\right)=f_{1}\left(S(u) u^{\prime} v, v\right), \quad u, u^{\prime} \in U\left(L^{-}+L_{0}\right) .
$$

Note that (2) ensures that $\lambda$ is well defined. We see from $(*)$ and (1) that $\lambda$ is a symmetric bilinear form which extends $f_{1}$. It is clear that $\lambda$ is $\left(L^{-}+L_{0}\right)$-associative. We claim that $\lambda$ is $L_{k}$-associative. To see this let $x \in L_{j}, y \in L_{b}$ and $z \in L_{k}$, where $-r \leqslant j, b \leqslant k$. We must show $\lambda([x, z], y)=\lambda(x,[z, y])$. Note that the result is trivially true unless $j+b=-r$. Then since $j, b \geqslant-r$ we may assume $j, b \leqslant 0$. As $\lambda$ is symmetric and $\left(L^{-}+L_{0}\right)$-associative we have

$$
\lambda([x, z], y)=-\lambda(z,[x, y])=-\lambda([x, y], z)=\lambda(x,[z, y]),
$$

as required. Thus $\lambda$ is $L_{k}$-associative and hence $L$-associative.

As $L$ is simple and $f$ is nondegenerate the nondegeneracy of $\lambda$ follows.

4. The associative forms of the graded Cartan type Lie algebras. Let $n$ be a positive integer and let $M=\left(m_{1}, \ldots, m_{n}\right)$ be an $n$-tuple. We consider the subalgebra $A(M, n)$ of the algebra of divided powers in $n$ variables which is generated as a vector space by the monomials $x^{(\alpha)}$, where $x^{(\alpha)}:=x_{1}^{\left(\alpha_{1}\right)} \cdots x_{n}^{\left(\alpha_{n}\right)}$ and $0 \leqslant \alpha_{i}<p^{m_{i}}$. For every $n$-tuple $\alpha$ we define $|\alpha|:=\sum_{i=1}^{n} \alpha_{i}$. In addition we order $n$-tuples by $\alpha \leqslant \beta$ iff $\alpha_{i} \leqslant \beta_{i} \forall i$. The letter $\tau$ will represent the $n$-tuple $\left(p^{m_{1}}-1, \ldots, p^{m_{n}}-1\right)$.

We will follow Kostrikin and Šafarevič [11] as regards notation. In this section we are going to determine those algebras among $W(M, n), S(M, n), H(M, n)$ and $K(M, n)$ that admit a nonsingular associative form. These simple, graded Lie algebras are known to be "transitively" graded, i.e. $\left[L_{-1}, L_{i}\right]=L_{i-1},-r \leqslant i \leqslant k$. In the following $F$ is assumed to be a field of positive characteristic $p$.

The following lemma shows in combination with Theorem 4.2 that simple Lie algebras which do not possess nondegenerate associative forms may very well admit $L^{-}$-associative forms.

LeMma 4.1. Let $\alpha_{\tau}: A(M, n) \rightarrow F$ be the linear map which is given by $\alpha_{\tau}\left(\sum_{0 \leqslant s \leqslant \tau} \beta_{s} x^{(s)}\right)=\beta_{\tau}$. Then the bilinear form $\lambda$ which is given by the equation

$$
\lambda\left(x^{(s)} \partial_{i}, x^{(t)} \partial_{j}\right)=\alpha_{\tau}\left(x^{(s)} x^{(t)}\right)
$$

is a $W(M, n)_{-1}$-associative form such that $\lambda\left(W(M, n)_{i}, W(M, n)_{j}\right)=0$ for $i+j \neq k$ -1 . 
Proof. The bilinear form $\lambda$ is obviously symmetric. Since

$$
\begin{aligned}
\lambda\left(\left[x^{(s)} \partial_{i}, \partial_{l}\right], x^{(t)} \partial_{j}\right) & =-\lambda\left(x^{\left(s-\varepsilon_{l}\right)} \partial_{i}, x^{(t)} \partial_{j}\right)=-(-1)^{\left|s-\varepsilon_{l}\right|} \alpha_{\tau}\left(x^{\left(s-\varepsilon_{l}+t\right)}\right) \\
& =(-1)^{|s|} \alpha_{\tau}\left(x^{\left(s-\varepsilon_{l}+t\right)}\right)=\lambda\left(x^{(s)} \partial_{i},\left[\partial_{l}, x^{(t)} \partial_{j}\right]\right)
\end{aligned}
$$

the assertion concerning the associativity follows. The particular property of $\lambda$ is a direct consequence of the definition.

THEOREM 4.2. The generalized Jacobson-Witt algebra $W(M, n)$ admits a nonsingular associative form if and only if $n=1$ and $p=3$ or $n=2$ and $p=2$.

Proof. We put $k:=\sum_{j=1}^{n}\left(p^{m_{j}}-1\right)-1$ and note that according to [11, p. 250] $W(M, n)=\oplus_{i=-1}^{k} W(M, n)_{i}$ is a simple, transitively graded Lie algebra. The algebra $H:=\left\langle\left\{x_{j} \partial_{j} ; 1 \leqslant j \leqslant n\right\}\right\rangle$ is a Cartan subalgebra of $W(M, n)_{0} \simeq \operatorname{gl}(n, F)$. For $0 \leqslant s \leqslant \tau$ we define linear forms $s: H \rightarrow F$ by means of $s\left(x_{j} \partial_{j}\right)=s_{j}$. Since $x^{(s)} \partial_{i} \in W(M, n)_{s-\varepsilon_{i}}$, where $\varepsilon_{i}=\left(\delta_{i j}\right)_{1 \leqslant j \leqslant n}$, we obtain

$$
W(M, n)=\sum_{i=1}^{n} \sum_{0 \leqslant s \leqslant \tau} W(M, n)_{s-\varepsilon_{i}} .
$$

Suppose that $W(M, n)$ admits a nonsingular associative form $\lambda$. By virtue of statement (3) of 3.2 we see that $W(M, n)_{k} \cap W(M, n)_{\varepsilon_{1}} \neq 0$. As $W(M, n)_{k} \subset$ $\bigoplus_{i=1}^{n} W(M, n)_{\tau-\varepsilon_{i}}$, we obtain $\varepsilon_{1}=\tau-\varepsilon_{i}$ for some $i$. This entails $n=1$ and $p=3$ or $n=2$ and $p=2$.

Now assume that $n=1$ and $p=3$. Consider the bilinear form $\lambda$ of 4 .1. We have

$$
\lambda\left(x^{(i)} \partial, x^{(j)} \partial\right)=(-1)^{i} \delta\left(i+j, p^{m}-1\right) .
$$

By virtue of our introductory remarks it will be sufficient to prove that $\lambda$ is $\left(W(M, 1)_{-1} \oplus W(M, 1)_{k}\right)$-associative. The $W(M, 1)_{-1}$-associativity follows from 4.1. Since $\lambda$ is symmetric and $\lambda\left(W(M, 1)_{i}, W(M, 1)_{j}\right)=0$ for $i+j \neq k-1$, it will suffice to verify the equation

$$
\lambda\left(\left[x \partial, x^{\left(p^{m}-1\right)} \partial\right], \partial\right)=\lambda\left(x \partial,\left[x^{\left(p^{m}-1\right)} \partial, \partial\right]\right) .
$$

The left-hand side is equal to -2 , while the right-hand side gives 1 . As $p=3$, we are done.

Finally, we assume $n=2$ and $p=2$. Consider the bilinear form $f: W(M, 2)_{-1} \times$ $W(M, 2)_{k} \rightarrow F$ which is given by $f\left(\partial_{i}, x^{(\tau)} \partial_{j}\right)=i+j, i, j \leqslant 2$. Then $f$ is nondegenerate and an elementary computation reveals that $f$ is $W(M, 2)_{0}$-associative. Noting that condition $(*)$ of 3.4 is linear in $u$, we see that we may assume that $u=$ $\partial_{1}^{p^{m 1}-1} \partial_{2}^{p^{m_{2}}-1}$. Since $S(u)=u$ and $u x^{(\tau)} \partial_{j}=\partial_{j},(*)$ follows. We now apply 3.4 in order to conclude the proof of our theorem.

REMARK. The following result is a generalization of a theorem due to $\mathrm{R}$. Block who proved the assertion in the restricted case (cf. [1]).

TheOREM 4.3. Suppose that $n \geqslant 3$. Then the special algebra $S(M, n)$ possesses $a$ nonsingular associative form if and only if $n=3$.

Proof. According to Proposition 1 on p. 254 of [11] $S(M, n)$ is a simple, transitively graded Lie algebra. We write $S(M, n)=\oplus_{i=-1}^{k} S(M, n)_{i}$ with $k:=\sum_{i=1}^{n}\left(p^{m_{i}}-1\right)-2$. Note that $S(M, n)_{-1}=W(M, n)_{-1}=\left\langle\left\{\partial_{1}, \ldots, \partial_{n}\right\}\right\rangle$. 
Hence $\operatorname{dim}_{F} S(M, n)_{-1}=n$. It can be readily verified that

$$
\left\{D_{i j}\left(x^{(\tau)}\right) ; 1 \leqslant i<j \leqslant n\right\}, \quad D_{i j}\left(x^{(s)}\right)=\partial_{j}\left(x^{(s)}\right) \partial_{i}-\partial_{i}\left(x^{(s)}\right) \partial_{j},
$$

is a basis of $S(M, n)_{k}$. Consequently, $\operatorname{dim}_{F} S(M, n)_{k}=n(n-1) / 2$. Suppose that $S(M, n)$ possesses a nonsingular associative form. By virtue of 3.2 we obtain

$$
n=\operatorname{dim}_{F} S(M, n)_{-1}=\operatorname{dim}_{F} S(M, n)_{k}=n(n-1) / 2 .
$$

Hence $n=3$.

In order to prove the existence of an associative form in case of $n=3$, we first recall the following formulas:

$$
\begin{aligned}
{\left[D_{i j}\left(x^{(a)}\right),\right.} & \left.D_{i k}\left(x^{(b)}\right)\right]=\left(\begin{array}{c}
a+b-\varepsilon_{i}-\varepsilon_{j} \\
a-\varepsilon_{j}
\end{array}\right) D_{i k}\left(x^{\left(a+b-\varepsilon_{i}-\varepsilon_{j}\right)}\right) \\
& +\left(\begin{array}{c}
a+b-2 \varepsilon_{i} \\
a-\varepsilon_{i}
\end{array}\right) D_{k j}\left(x^{\left(a+b-2 \varepsilon_{i}\right)}\right)+\left(\begin{array}{c}
a+b-\varepsilon_{i}-\varepsilon_{k} \\
a-\varepsilon_{i}
\end{array}\right) D_{j i}\left(x^{\left(a+b-\varepsilon_{i}-\varepsilon_{h}\right)}\right) .
\end{aligned}
$$

For any mapping $\sigma:\{1, \ldots, n\} \rightarrow\{1, \ldots, n\}$ we define the signature

$$
\operatorname{sgn}(\sigma):=\prod_{i<j} \frac{\sigma(i)-\sigma(j)}{i-j} .
$$

Note that $\operatorname{sgn}(\sigma)=0$ if $\sigma$ is not a permutation. We define a bilinear mapping $f$ : $S(M, 3)_{-1} \times S(M, 3)_{k} \rightarrow F$ by means of

$$
f\left(\partial_{l}, D_{i j}\left(x^{(\tau)}\right)\right)=\operatorname{sgn}(l, i, j) .
$$

In order to show that $f$ is $S(M, 3)_{0}$-associative we will have to verify the equation

(3) $f\left(\left[\partial_{q}, D_{l k}\left(x^{(b)}\right)\right], D_{i j}\left(x^{(\tau)}\right)\right)=f\left(\partial_{q},\left[D_{l k}\left(x^{(b)}\right), D_{i j}\left(x^{(\tau)}\right)\right]\right)$ for $|b|=2$.

We may assume that $l \neq k$ and $i \neq j$. Hence we have $\{i, j\} \cap\{l, k\} \neq \varnothing$. As $D_{l k}\left(x^{(b)}\right)=-D_{k l}\left(x^{(b)}\right)$ and $D_{j i}\left(x^{(\tau)}\right)=-D_{i j}\left(x^{(\tau)}\right)$ we may assume without loss of generality that $l=i$. Using (1) we see that (3) is equivalent to

$$
-f\left(x^{\left(b-\varepsilon_{i}-\varepsilon_{q}\right)} \partial_{k}, D_{i j}\left(x^{(\tau)}\right)\right)=f\left(\partial_{q},\left[D_{i k}\left(x^{(b)}\right), D_{i j}\left(x^{(\tau)}\right)\right]\right) .
$$

Formula (2) now shows that both sides of (4) vanish unless $b_{i} \geqslant 1$. Hence we may assume that $b=\varepsilon_{i}+\varepsilon_{i}$.

In that case (2) implies

$$
\left[D_{i k}\left(x^{(b)}\right), D_{i j}\left(x^{(\tau)}\right)\right]=\delta_{k t} D_{j i}\left(x^{(\tau)}\right)+\delta_{i t} D_{k j}\left(x^{(\tau)}\right)+\delta_{j t} D_{i k}\left(x^{(\tau)}\right) .
$$

Consequently, we obtain

$f\left(\partial_{q},\left[D_{i k}\left(x^{(b)}\right), D_{i j}\left(x^{(\tau)}\right)\right]\right)=\delta_{k t} \operatorname{sgn}(q, j, i)+\delta_{i t} \operatorname{sgn}(q, k, j)+\delta_{j t} \operatorname{sgn}(q, i, k)$.

If $k=j$, then both sides of (4) are readily seen to vanish. Otherwise $|\{i, j, k\}|=3$ and the right-hand side of (4) equals

$$
\begin{aligned}
\delta_{k t} \delta_{q k} \operatorname{sgn}(k, j, i)+\delta_{i t} \delta_{q i} & \operatorname{sgn}(i, k, j)+\delta_{j t} \delta_{q j} \operatorname{sgn}(j, i, k) \\
& =-\operatorname{sgn}(k, i, j) \delta_{q t}\left(\delta_{q k}+\delta_{q i}+\delta_{q j}\right)=-\operatorname{sgn}(k, i, j) \delta_{q t} .
\end{aligned}
$$

The last expression, however, coincides with the left-hand side of (4). 
Since $f$ is clearly nondegenerate, we may conclude the proof by verifying condition $(*)$ of 3.4. For $s=\left(s_{1}, s_{2}, s_{3}\right)$ we put $\partial^{s}:=\partial_{1}^{s_{1}} \partial_{2}^{s_{2}} \partial_{3}^{s_{3}}$. The P-B-W Theorem then implies that it suffices to check $(*)$ for $u_{l}:=\partial^{\tau-\varepsilon_{l}}$.

Using (1) we obtain $u_{l} D_{i j}\left(x^{(\tau)}\right)=D_{i j}\left(x^{\left(\varepsilon_{l}\right)}\right)=\delta_{j l} \partial_{i}-\partial_{i l} \partial_{j}$ while $S\left(u_{l}\right)=-u_{l}$. Condition (*) now follows from the succeeding identities:

$$
\begin{aligned}
f\left(u_{l} D_{i j}\left(x^{(\tau)}\right), D_{i k}\left(x^{(\tau)}\right)\right) & =-\delta_{i l} \operatorname{sgn}(j, i, k) \\
& =\delta_{i l} \operatorname{sgn}(k, i, j)=f\left(S\left(u_{e}\right) D_{i k}\left(x^{(\tau)}\right), D_{i j}\left(x^{(\tau)}\right)\right) .
\end{aligned}
$$

Throughout the remainder of this paper we assume $p>2$.

We proceed by recalling some basic facts pertaining to the hamiltonian algebra $H(M, 2 r)$. For $j \in\{1, \ldots, 2 r\}$ we put

$$
j^{\prime}:= \begin{cases}j+r, & 1 \leqslant j \leqslant r \\ j-r, & r+1 \leqslant j \leqslant 2 r\end{cases}
$$

as well as

$$
\sigma(j):= \begin{cases}1, & 1 \leqslant j \leqslant r \\ -1, & r+1 \leqslant j \leqslant 2 r .\end{cases}
$$

For $f \in A(M, 2 r)$ we define $D_{H}(f)$ by means by

$$
D_{H}(f):=\sum_{j=1}^{2 r} \sigma(j) \partial_{j}(f) \partial_{j^{\prime}} .
$$

It is well known (cf. [11, 13]) that $B:=\left\{D_{H}\left(x^{(s)}\right) ; 0<s<\tau\right\}$ is a basis of $H(M, 2 r)$ over $F$. We also recall the formula

$$
\left[D_{H}(f), D_{H}(g)\right]=D_{H}\left(D_{H}(f)(g)\right) \quad \forall f, g \in A(M, 2 r) .
$$

The proof of the following result was communicated to the author by $H$. Strade. The result was first proved by Block in a special case (cf. [1]).

THEOREM 4.4. The hamiltonian algebra $H(M, 2 r)$ possesses a nonsingular associative form.

Proof. Consider the linear map $\alpha_{\tau}: A(M, 2 r) \rightarrow F$ which has been defined in the course of the proof of 4.2. We define a bilinear form by means of

$$
f\left(D_{H}\left(x^{(s)}\right), D_{H}\left(x^{(t)}\right)\right):=\alpha_{\tau}\left(x^{(s)} x^{(t)}\right) .
$$

The form $f$ is obviously symmetric and nontrivial. By virtue of (4.3.1) we have

$$
D_{H}\left(D_{H}\left(x^{(s)}\right)\left(x^{(t)}\right)\right)=\left[D_{H}\left(x^{(s)}\right), D_{H}\left(x^{(t)}\right)\right] \in D_{H}\left(\bigoplus_{s<\tau} F x^{(s)}\right) .
$$

Note that

$$
D_{H}\left(x^{(s)}\right)\left(x^{(t)} x^{(r)}\right)=D_{H}\left(x^{(s)}\right)\left(x^{(t)}\right) x^{(r)}+x^{(t)} D_{H}\left(x^{(s)}\right)\left(x^{(r)}\right) .
$$

Since $\operatorname{ker} D_{H}=F_{1}(4.3 .2)$ yields

and we therefore obtain

$$
\left(\begin{array}{c}
t+r \\
t
\end{array}\right) \alpha_{r}\left(D_{H}\left(x^{(s)}\right)\left(x^{(t+r)}\right)\right)=0
$$

$$
\alpha_{\tau}\left(D_{H}\left(x^{(s)}\right)\left(x^{(t)}\right) x^{(r)}\right)=-\alpha_{\tau}\left(D_{H}\left(x^{(s)}\right)\left(x^{(r)}\right) x^{(t)}\right) .
$$


This shows that

$$
\begin{aligned}
f\left(\left[D_{H}\left(x^{(s)}\right)\right.\right. & \left.\left., D_{H}\left(x^{(t)}\right)\right], D_{H}\left(x^{(r)}\right)\right)=\alpha_{\tau}\left(D_{H}\left(x^{(s)}\right)\left(x^{(t)}\right) x^{(r)}\right) \\
& =-\alpha_{\tau}\left(D_{H}\left(x^{(s)}\right)\left(x^{(r)}\right) x^{(t)}\right)=\alpha_{\tau}\left(D_{H}\left(x^{(r)}\right)\left(x^{(s)}\right) x^{(t)}\right) \\
& =-\alpha_{\tau}\left(D_{H}\left(x^{(r)}\right)\left(x^{(t)}\right) x^{(s)}\right)=-f\left(\left[D_{H}\left(x^{(r)}\right), D_{H}\left(x^{(t)}\right)\right], D_{H}\left(x^{(s)}\right)\right) \\
& =f\left(D_{H}\left(x^{(s)}\right),\left[D_{H}\left(x^{(t)}\right), D_{H}\left(x^{(r)}\right)\right]\right) .
\end{aligned}
$$

Hence $f$ is associative.

The contact algebra $K(M, 2 r+1)$ is defined by means of the mapping

$$
D_{K}:=\left\{\begin{array}{l}
A(M, 2 r+1) \rightarrow W(M, 2 r+1), \\
f \rightarrow D_{H}(f)+\sum_{j=1}^{2 r} x_{j} D_{j 2 r+1}(f)+2 f \partial_{2 r+1} .
\end{array}\right.
$$

For $f, g \in A(M, 2 r+1)$ we put

$$
\langle f, g\rangle:=D_{K}(f)(g)-2 g \partial_{2 r+1}(f) \text {. }
$$

It is well known that

$$
D_{K}(\langle f, g\rangle)=\left[D_{K}(f), D_{K}(g)\right]
$$

We define

$$
K(M, 2 r+1):= \begin{cases}\sum_{s<\tau} F x^{(s)}, & n+3 \equiv 0 \bmod (p), \\ A(M, 2 r+1), & \text { otherwise. }\end{cases}
$$

We put $\|s\|:=|s|+s_{2 r+1}-2$. Let $K(M, n)_{i}:=\left\langle\left\{x^{(s)} ;\|s\|=i\right\}\right\rangle$ where $n=2 r+1$. Then $K(M, n):=\bigoplus_{i=-2}^{k} K(M, n)_{i}$ is a simple, transitively and admissibly graded Lie algebra with

$$
k:= \begin{cases}\sum_{i=1}^{n}\left(p^{m_{i}}-1\right)+p^{m_{n}}-4, & n+3 \equiv 0 \bmod (p), \\ \sum_{i=1}^{n}\left(p^{m_{i}}-1\right)+p^{m_{n}}-3, & \text { otherwise. }\end{cases}
$$

THEOREM 4.5. The contact algebra $K(M, n)$ possesses a nonsingular associative form if and only if $n+5 \equiv 0 \bmod (p)$.

Proof. Noting that $K(M, n)_{0}=\left\langle\left\{x_{i} x_{j} ; 1 \leqslant i, j \leqslant n\right\} \cup\left\{x_{n}\right\}\right\rangle$ we see that

$$
H_{0}:=\sum_{j=1}^{r} F x_{j} x_{j}+F x_{n}
$$

is a Cartan subalgebra of $K(M, n)_{0}$ which operates on

$$
K(M, n)_{-1}:=\left\langle\left\{x_{1}, \ldots, x_{n-1}\right\}\right\rangle
$$

by semisimple endomorphisms. The definition of the multiplication gives rise to

$$
\left\langle x_{n}, x^{(t)}\right\rangle=\|t\| x^{(t)} .
$$

Consequently, $\Omega:=C_{K(M, n)}\left(H_{0}\right)$ is contained in $\bigoplus_{i \equiv 0 \bmod (p)} K(M, n)_{i}$. 
Suppose that $K(M, n)$ has a nonsingular associative form. Then 3.2 ensures that $\Omega$ intersects $K(M, n)_{k-2}$ and we obtain $k-2 \equiv 0 \bmod (p)$. The condition stated in the theorem is therefore necessary.

Now assume conversely that $n+5 \equiv 0 \bmod (p)$. Then $K(M, n)=\oplus_{s \leqslant \tau} F x^{(s)}$. We define a bilinear form $f$ on $K(M, n)_{-1} \times K(M, n)_{k}$ by means of $f\left(1, x^{(\tau)}\right):=1$. The condition $n+5 \equiv 0 \bmod (p)$ ensures that $f\left(\left\langle 1, x_{n}\right\rangle, x^{(\tau)}\right)=f\left(1,\left\langle x_{n}, x^{(\tau)}\right\rangle\right)$. The equation $f\left(\left\langle 1, x_{i}, x_{j}\right\rangle, x^{(\tau)}\right)=f\left(1,\left\langle x_{i} x_{j}, x^{(\tau)}\right\rangle\right)$ follows from the identities

$$
\begin{gathered}
\left\langle 1, x^{(t)}\right\rangle=2 x^{\left(t-\varepsilon_{n}\right)}, \\
\left\langle x_{i} x_{j}, x^{(\tau)}\right\rangle=0 .
\end{gathered}
$$

In order to verify $(*)$ of 3.4 we note that

$$
\left\langle x_{j}, x^{(t)}\right\rangle=\sigma(j) x^{\left(t-\varepsilon_{j^{\prime}}\right)}+\left(t_{j}+1\right) x^{\left(t-\varepsilon_{n}+\varepsilon_{j}\right)}, \quad 1 \leqslant j \leqslant n-1 .
$$

Let $y^{s}:=x_{1}^{s_{1}} \cdots x_{n-1}^{s_{n-1}} 1^{s_{n}}$ in $U\left(K(M, n)^{-}\right)$. The P-B-W Theorem then shows that $(*)$ only needs to be verified for $u:=y^{\tau}$. Note that $1^{p^{m n-1}} x^{(\tau)}=x^{(a)}$ with $a_{n}=0$. It is then easy to see that $u x^{(\tau)}=1$. Since $S(u)=x_{n-1}^{p^{m_{n-1}}-1} \cdots x_{1}^{p^{m_{1}}-1} 1^{p^{m_{n}}-1}$ the same reasoning proves that $S(u) x^{(\tau)}=1$. This establishes $(*)$.

REMARK. One can show that the uniquely determined associative form $\lambda$ extending $f$ is given by $\lambda\left(x^{(s)}, x^{(t)}\right)=\alpha_{\tau}\left(x^{(s)} x^{(t)}\right)$.

\section{BIBLIOGRAPHY}

1. R. E. Block, New simple Lie algebras of prime characteristic, Trans. Amer. Math. Soc. 89 (1958), 421-449.

2. __ On Lie algebras of classical type, Proc. Amer. Math. Soc. 11 (1960), 377-379.

3. _ Trace forms on Lie algebras, Canad. J. Math. 14 (1962), 553-564.

4. On the extensions of Lie algebras, Canad. J. Math. 20 (1968), 1439-1450.

5. R. Cartier, Séminaire "Sophus Lie" (Faculté Sci. Paris, 1955/56), Secrétariat Math., Paris, 1957.

6. M. Ju. Celousov, Derivations of Lie algebras of Cartan type, Izv. Vysš. Učebn. Zaved. Matematika 7 , $126-134$.

7. R. Farnsteiner, On Ad-semisimple Lie algebras, J. Algebra 83 (1983), 510-519.

8. M. Frank, Restrictedness and simplicity in Lie algebras of characteristic $p$, Scripta Math. 29 (1973), 395-407.

9. G. P. Hochschild, Cohomology of restricted Lie algebras, Amer. J. Math. 76 (1954), 555-580.

10. I. Kaplansky, Lie algebras and locally compact groups, Chicago Lecture Notes, 1974.

11. A. I. Kostrikin and I. R. Safarevic, Graded Lie algebras of finite characteristic, Math. USSR Izv. 3 (1969), 237-304.

12. G. B. Seligman, Modular Lie algebras, Ergebnisse der Math., vol. 40, Springer, Berlin and New York, 1967.

13. R. L. Wilson, A structural characterization of the simple Lie algebras of generalized Cartan type over fields of prime characteristic, J. Algebra 40 (1976), 418-465.

14. D. J. Winter, On groups of automorphisms of Lie algebras, J. Algebra 8 (1968), 131-142.

Department of Mathematics, University of California, Riverside, California 92521 